\title{
DA-DA Düşürücü Dönüştürücünün Sistem Tanımlaması ve Kontrolü
}

\author{
Davut Sevim ${ }^{1 *}$, Nurullah Bozkurt ${ }^{2}$ \\ 1* Batman Üniversitesi, Mühendislik Mimarlık Fakültesi, Elektrik Elektronik Mühendisliği Bölümü, Batman, Türkiye, (ORCID: 0000-0002-0227-4772), \\ davut.sevim@batman.edu.tr \\ ${ }^{2}$ Batman Üniversitesi, Lisansüstü Eğitim Enstitüsü, Elektrik Elektronik Mühendisliği Bölümü, Batman, Türkiye (ORCID: 0000-0002-6932-5327), \\ nurullah.bozkurt@batman.edu.tr
}

(Illk Geliş Tarihi 5 Kasım 2021 ve Kabul Tarihi 29 Aralık 2021)

(DOI: 10.31590/ejosat.1019650)

\begin{abstract}
ATIF/REFERENCE: Sevim, D., \& Bozkurt, N. (2021). DA-DA Düşürücü Dönüştürücünün Sistem Tanımlaması ve Kontrolü. Avrupa Bilim ve Teknoloji Dergisi, (31), 881-889.

$\ddot{O} \mathbf{z}$

Güç elektroniği uygulamalarından biri olan düşürücü tip DA-DA dönüştürücüler, doğrusal olmadıklarından dolayı transfer fonksiyonu elde etmede uygun bir metot ile tanımlanma ihtiyacı duyarlar. Bu dönüştürücünün transfer fonksiyonunu elde etmek için matematiksel modelleme gibi karmaşık işlemler gerekmektedir. Bu sıkıntıları ortadan kaldıran yöntemlerden bir tanesi Black-box tabanlı sistem tanımlama yöntemidir. Bu çalışmada, Black-box tabanlı DA-DA düşürücü dönüştürücü tasarımı gerçekleştirilmiştir. Tasarımı yapılan sistemin, transfer fonksiyonu katsayıları belirlenmiştir. Önerilen modelin sisteme hangi oranda benzediği ve performans değerleri çıkarılmıştır. Sistemin daha kararlı bir hale getirilmesi için PID kontrolör eklenerek, kontrolör parametreleri belirlenmiştir. Tasarlanan sistemin, kontrollü ve kontrolsüz durumlardaki açık çevrim ve kapalı çevrim performansları kıyaslanmıştır. Devre sonuçları incelendiğinde, Black-box yöntemi ile çıkarımı yapılan transfer fonksiyonunun devreyi hızlı ve doğru bir şekilde modellediği ve kontrolör tasarımının basitleştirildiği görülmüştür.
\end{abstract}

\section{System Identification and Control of DC-DC Step-Down Converter}

\begin{abstract}
Buck converters are forming one of the applications of power electronics. As they have non-linear structure, an appropriate method is required in order to obtain the transfer function. In terms of obtaining the transfer function, complex operations, including mathematical modeling, are required. The black-box-based system identification is one of the methods that can ease the problems. Therefore, in this study, black-box based buck converter design has been realized. The transfer function coefficients of the designed system were determined. The performance values were extracted with the proposed model. In order to make the system more stable, the PID controller was added and the controller parameters were determined. The open-loop and closed-loop performances of the designed system in controlled and uncontrolled cases were compared. It was seen that the transfer function extracted by the Black-box method simplifies the controller design and quickly and accurately obtains the circuit model.
\end{abstract}

Keywords: System identification, Buck converter, Black-Box, Transfer function.

\footnotetext{
*Sorumlu Yazar: davut.sevim@batman.edu.tr
} 


\section{Giriş}

Yarı iletken teknolojisindeki gelişmeler ile güç elektroniğinde çok ciddi gelişmeler meydana gelmiştir. Günümüzde güç elektroniğgi sistemleri, birçok endüstriyel uygulamada yaygın olarak kullanılmaktadır. Bu uygulamalardan biri de anahtarlamalı Düşürücü tip DA-DA (Doğru Akım-Doğru Akım) dönüştürücüleridir. Düşürücü tip DA-DA dönüştürücüler, işletmelerde üretimi gerçekleştiren makinelerin güç kaynaklarında, motor kontrol devrelerinde ve günlük hayatta kullanılan bilgisayar, televizyon gibi birçok üründe kullanılmaktadır (Yalduz, 2015).

Düşürücü tip DA-DA dönüştürücüler bobinli olurlar ve genellikle izolesiz yapıda üretilirler. Bu dönüştürücüler temelde giriş gerilim kaynağı ve yük hariç iki aktif ve iki pasif devre elemanının çalışma devresine bağlanması ile elde edilirler (Rashid, 2007). Düşürücü tip DA-DA dönüştürücülerde, çıkış voltajının büyüklüğü, dönüştürücü anahtarının girişindeki PWM (Darbe Genişlik Modülasyonu) sinyalinin görev oranının kontrol edilmesi ile sağlanır (Özdemir ve Yazıcı, 2018; Emadi, 2005).

Özellikle, anahtarlamalı dönüştürücülerin doğrusal olmayan yapısından dolayı, tam matematiksel tanımlarını kullanarak kararlı geri besleme kontrolörlerini tasarlamak çok zordur. Genellikle, anahtarlamalı dönüştürücüler doğrusal olmayan diferansiyel denkleme sahiptir ve çözümlemesi karmaşıktır (Choi, 1998). Bu sebepten dolayı transfer fonksiyonu çıkarımı gibi uygun bir metot ile tanımlanmaları gerekmektedir. Transfer fonksiyonu çıkarımı için matematiksel modelleme gibi nispeten karmaşık görünen tekniğin yanında sistem tanımlama yöntemleri de kullanılmaktadır. Bu yöntemlerden biri de Black-box tabanlı yöntemdir. Bu yöntemde sistemin giriş/çıkış değerleri ölçülür ve sistemi temsil eden bir model önerilir. Black-box yöntemi sayesinde bir dönüştürücünün dinamik davranışını tanımlayan transfer fonksiyonu ve benzeri modeller önerilebilir.

Jin-Hong vd. DA-DA dönüştürücü sisteminin bir parametre tahminini ele almaktadır. DA-DA dönüştürücü sisteminin teşhisi için ana parametrenin sistem tanımlama yöntemi ile tahmin edilebileceğini ve sistemin yaşlanma durumunun, çalışma durumu üzerindeki bu sonuçlarla tahmin edilebileceğini doğrulamaktadır (Jin-Hong, Tae-Jin, Kwang-Su ve Kwang-Hwa, 2004). Buatti vd. DA-DA dönüştürücünün parametrelerini tanımlamak için en küçük kareler yöntemini kullanan bir yöntem sunmuştur (Buiatti, Amaral ve Marques Cardoso, 2007). Zhao, basit donanımla uygulanabilen otomatik ayarlı kontrolör geliştirmiştir (Zhao, 2008). Kontrolör, kasıtlı olarak getirilen sınır döngüsü salınımlarının genliğini ve frekansını inceleyerek çıkış kapasitansı, yük direnci, köşe frekansı ve sönümleme faktörü gibi güç aşaması parametrelerini tahmin etmiştir. Algreer vd. DA-DA dönüştürücünün parametrelerini tanımlamak için yeni bir çevrimiçi yaklaşım önermişlerdir. Önerilen yöntem, geçici dönemlerde ani yük değişikliklerini hızlı bir şekilde saptayarak hesaba katmıştır. Bununla birlikte tahmin algoritması, çıktı tepe aşım değerine ulaşmadan önce model parametrelerini güncelleyebilmiştir (Algreer, Armstrong ve Giaouris, 2009). Osman vd. zaman alanına dayalı bir sistem tanımlama yöntemi geliştirmişlerdir. Örneklenen veriler, matematiksel bir modeli tahmin etmek ve doğrulamak için analiz ederek ön işleme tabi tutmuştur. Önerilen yöntem, yük parametresini iyi bir doğrulukla belirleyebilmiştir (Osman \& Osman, 2011). Taborda vd. hata üzerinden Sıfır Ortalama Dinamik veya ZAD (Sifir Ortalama Dinamikler) stratejisine dayalı Dijital-PWM kontrollü düşürücü e-ISSN: 2148-2683 dönüştürücüde yeni bir parametre tahmin yöntemi bildirilmiştir (Taborda, Angulo ve Olivar, 2011). Li vd. DA-DA düşürücü dönüştürücünün tam parametre tahmini için yeni bir yaklaşım önermiştir. Yeni yaklaşım, biyocoğrafya tabanlı optimizasyon kullanılarak gerçekleştirilmiştir. Geleneksel RLS (Öz Yinelemeli En küçük Kareler) yönteminin aksine, önerilen yöntem tam durum gözlemli bir durum uzayı modeline dayandırılmaktadır. $\mathrm{Bu}$ nedenle, farklı ölçüm gürültü seviyeleri altında parametre tahmin performansı iyileştirilmiştir (Li ve Low, 2016). Cen vd. düşürücü dönüştürücülerin durumlarını izlemek ve kapasitans, endüktans gibi bileşen parametrelerini tahmin etmek için model gözlemci tabanlı bir şema önermişlerdir (Cen ve Stewart, 2017). Abbas vd. dönüştürücülerin dinamiklerinin tahminini ele almışlar. Önerilen araştırmada, dönüştürücü dinamiklerini tahmin etmek için görev döngüsünden çıkış voltajı tahminine kadar frekans cevabına dayalı veriler kullanmıştır (Abbas ve Abbas, 2018). Padhee vd. düşürütü tip DA-DA dönüştürücü için kapalı döngü parametrik sistem tanımlamasının adım adım analizini sağlamıştır. Kapalı döngü parametrik tanımlamada, giriş-çıkış deneysel verileri, düşürücü tip DA-DA dönüştürücünün transfer fonksiyonu katsayılarını tahmin etmek için kullanmıştır (Padhee, Pati, ve Mahapatra, 2018). Andries vd. yazılım kontrol döngüsüne sahip bir düşürücü tip DA-DA dönüştürücüsüne uygulanan ayrı bir zaman alanlı sistem tanımlama yöntemini açıklamaktadır. Yöntemin özü, düşürücü tip DA-DA dönüştürücünün, PWM kontrol sinyalinin girdisi olarak dikkate alınarak dönüştürücünün çıkışının açık döngü dinamiklerine iyi bir doğrulukla yaklaşan bir transfer fonksiyonunun tahmin edilmesinden oluşur (Andries, Goras, David, Buzo ve Pelz, 2019).

$\mathrm{Bu}$ makalede, bir düşürücü tip DA-DA dönüştürücü tasarımının Simulink ortamında gerçekleştirilmesi amaçlanmıştır. Tasarım için sistemin transfer fonksiyonu elde edilmeye çalışılmıştır. Düşürücü tip DA-DA dönüştürücünün transfer fonksiyonunu elde etmek için sistem tanımlama yöntemlerinden biri olan Black-box sistem tanımlama modeli önerilmiştir. Giriş ve çıkış değerleri tanıtılmış ve Black-box tabanlı sistem tanımlama yöntemi kullanılarak, tasarımı yapılan sistemin transfer fonksiyonu katsayıları belirlenmiştir. Katsayılar belirlenirken önerilen modellerin, sisteme hangi oranda benzediği belirlenerek performansları ortaya çıkarılmıştır. Buradan kontrolsüz bir şekilde tasarımı yapılan simülasyon devresinin aşım miktarını azaltmak ve sistemi daha kararlı bir hale getirmek için simülasyon devresine PID kontrolör eklenmiştir. Kontrolör parametrelerinin düzgün bir şekilde seçilmesi sistemin kararlılık durumunu yüksek derecede etkilemektedir. Kontrolör parametreleri belirlemek için Matlab arayüzünden faydalanılmıştır. Devre sonuçları incelendiğinde, Black-box yöntemi ile çıkarımı yapılan transfer fonksiyonunun devreyi hızlı ve doğru bir şekilde modelleyebildiği görülmüş aynı zamanda kontrolör tasarımı basitleştirilmiştir.

\section{DA-DA Düşürücü Tip Dönüştürücü Modeli Materyal ve Metot}

Düşürücü tip DA-DA dönüştürücü devresi temel olarak belirli bir DA giriş voltajını istediğimiz daha düşük bir DA çıkış voltajına dönüştürme işlevini gerçekleştirir. Devrede kontrol edilebilen bir yarı iletken anahtar, bir diyot, bir LC alçak geçiren filtre ve çıkışta bir yük bulunmaktadır.

Çıkış voltajını dönüştürme işlemi yarı iletken güç anahtarının kontrol edilmesi ile gerçekleştirilir. Kontrol işlemleri 
için çeşitli metotlar mevcuttur. Ancak son yıllarda güç elektroniği alanında yaygın olarak kullanılan kontrol tekniği PWM tekniğidir.

PWM kontrol yönteminde, referans gerilim ile çıkış gerilimi farkı yükseltilir. Elde edilen kontrol işareti, testere dişi dalga ile karşılaştırılır. Karşılaştırıcı devrenin çıkışından sisteme uygulanacak olan PWM kontrol işareti elde edilir (Bodur, 2014).

Şekil.1'de temel düşüren dönüştürücü devresi, görev döngüsü tanımı ve eşitliği verilmiştir.

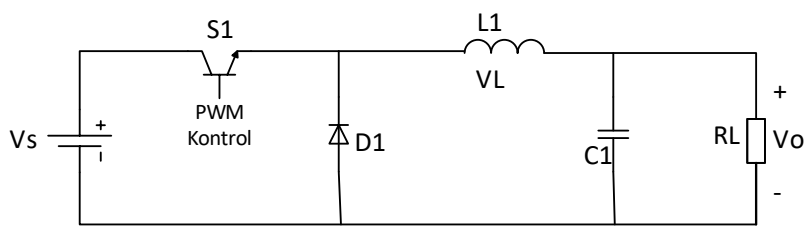

Şekil 1. Düşürücü tip DA-DA dönüştürücü devresi

Anahtarlama frekansı ve periyot arasındaki devre eşitliği;

$$
\mathrm{f}=\frac{1}{\mathrm{~T}}
$$

Bir periyot süresinde S1 anahtarının açık olduğu zamana $t_{\text {on }}$, kapalı olduğu zamana $t_{\text {off }}$ diye belirtilirse görev döngüsü veya doluluk oranı (duty cycle) anahtarlama elemanının iletimde olduğu sürenin tüm periyot süresine oranı olarak adlandırılır. Doluluk oranı aşağıdaki eşitlik ile tanımlanır.

$$
\mathrm{D}=\frac{\mathrm{t}_{\text {on }}}{\mathrm{t}_{\text {on }}+\mathrm{t}_{\text {off }}}=\frac{\mathrm{t}_{\text {on }}}{\mathrm{T}} \quad 0 \leq \mathrm{D} \leq 1
$$

İdeal bir dönüştürücüde tüm elemanlar mükemmel kabul edilir. Bobin ve Kondansatörün iç dirençleri ihmal edilir.

Düşürücü dönüştürücü sürekli iletim modunda (CCM) veya süreksiz iletim modunda (DCM) çalışabilir. İkisi arasındaki fark, sürekli iletim modunda bobindeki akımın sıfıra düşmemesidir. Genel performans açısından kıyaslanırsa sürekli iletim modunda maksimum çıkıș gücü elde edilir. Devredeki çalışma mantığının anlaşılabilmesi için S1 yarı iletken anahtarın açık ve kapalı olduğu durumlar ile ilgili devre elemanlarının akım ve gerilim değerleri incelenmelidir:

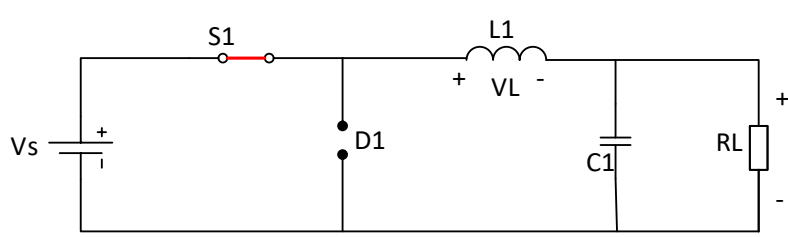

Şekil 2. Anahtarın iletim durumu

Anahtar iletim durumunda iken; diyot kesimde olur, giriş gerilim kaynağı anahtar açık olduğu sürece $t_{\text {on }}=\mathrm{DT}$ devre elemanlarını besler. Bobin gerilimi giriş gerilimi ile çıkış geriliminin farkına eşit olur. Kondansatör gerilimi de çıkış gerilimine eşitlenir.

$$
\begin{aligned}
& \mathrm{V}_{\mathrm{L}}=\mathrm{V}_{\mathrm{S}}-\mathrm{V}_{0} \\
& \mathrm{~V}_{\mathrm{C}}=\mathrm{V}_{0}
\end{aligned}
$$

Bobin gerilimi doğrusal bir şekilde artar ve bobinde bir miktar enerji birikir. Bobin geriliminin akıma bağlı değişimi;

$$
V_{L}(t)=L \frac{d i_{L}(t)}{d(t)}
$$

2.3, 2.4 ve 2.5 eşitliklerinden;

$$
\begin{aligned}
& V_{L}=V_{S}-V_{0}=L \frac{d i_{L}(t)}{d(t)} \\
& \frac{d i_{L}(t)}{d(t)}=\frac{V_{S}-V_{0}}{L} \\
& i_{C}=C \frac{d V_{0}(t)}{d(t)} \\
& \frac{d V_{0}(t)}{d(t)}=\frac{1}{C}\left(i_{L}-\frac{V_{0}}{R}\right)
\end{aligned}
$$

Anahtar açık durumdaki (2.6), (2.7), (2.8) ve denklemleri elde edilir.

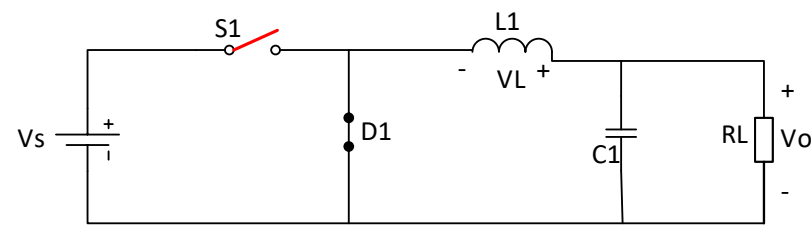

Şekil 3. Anahtarın kesim durumu

Anahtar kesim durumunda iken; diyot iletimde olur ve giriş kaynak gerilimi devre dışı kalır. Anahtar kapalı olduğu sürece $\mathrm{t}_{\text {off }}=(1-\mathrm{D}) \mathrm{T}$ bobin deşarj olmaya başlar, voltaj polaritesi tersine döner, bobin üzerindeki yük kondansatör üzerinden akmaya başlar. Bobin gerilimi ve kondansatör gerilimi çıkış gerilimine eşitlenir.

$$
\begin{aligned}
& -V_{0}=L \frac{d i_{L}(t)}{d(t)} \\
& \frac{d i_{L}(t)}{d(t)}=-\frac{V_{0}}{L} \\
& \frac{d V_{0}(t)}{d(t)}=\frac{1}{C}\left(\frac{i_{L}-V_{0}}{R}\right)
\end{aligned}
$$

Anahtar kapalı durumdaki (2.10), (2.11) ve (2.12) denklemleri elde edilir. Anahtar açık ve kapalı durumları bobin gerilimi üzerine uygulanırsa;

$$
\begin{aligned}
& V_{\text {Lon }} t_{\text {on }}+V_{\text {Loff }} t_{\text {off }}=0 \\
& \left(V_{S}-V_{0}\right) D T+\left(-V_{0}\right)(1-D) T=0 \\
& V_{0}=D V_{S} \\
& D=\frac{V_{0}}{V_{S}}
\end{aligned}
$$

Düşürücü dönüştürücü doluluk oranı denklemi (2.16) elde edilir. Söz konusu denklem sonucunda görüleceği üzere çıkış voltajı giriş voltajına ve doluluk oranına doğrudan bağlıdır.

\section{Sistem Tanımlama ile Transfer Fonksiyonu Çözümü}

\subsection{Transfer Fonksiyonu}

Transfer fonksiyonu modelleri, bir polinom oranını kullanarak bir sistemin girdileri ve çıktıları arasındaki ilişkiyi tanımlar. Payda polinomunun köklerine model kutuplar denir. Pay polinomunun köklerine model sifirlar denir. Bir transfer fonksiyonu modelinin parametreleri, kutupları, sıfirları ve iletim gecikmeleridir. Sürekli zamanda, bir transfer fonksiyonu modeli şu biçime sahiptir:

$Y(s)=\frac{\operatorname{num}(s)}{\operatorname{den}(s)} U(s)+E(s)$ 
Burada, $\mathrm{Y}(\mathrm{s}), \mathrm{U}(\mathrm{s})$ ve $\mathrm{E}(\mathrm{s})$ sırasıyla çıkış, giriş ve gürültünün Laplace dönüşümlerini temsil eder. num(s) ve den(s), girdi ve çıktı arasındaki ilişkiyi tanımlayan pay ve payda polinomlarını temsil eder (Mathworks., 2021).

Düşürücü dönüştürücü devresinin transfer fonksiyonunun çıkarımı için Kirchhoff akım ve gerilim yasalarından faydalanılır (Osman, 2011).

Devre denklemleri çözümlendikten sonra düşürücü dönüştürücü devresi transfer fonksiyonu aşağıdaki gibi elde edilir (Pembegüllü, 2019);

$G_{v d}(s)=\frac{V_{0}(s)}{V_{s}(s)}=\frac{1}{s^{2} L C+s \frac{L}{R}+1}$

\subsection{Sistem Tanımlama}

Sistem tanımlama, dinamik sistemlerin matematiksel modellerini oluşturmak için ölçülen verilerden istatistiksel yöntemler kullanan bir yaklaşım sunar (Goodwin, 1977). Sistem tanımlamadaki amaç sistemin davranışının ve dış etkilerin (sisteme girdiler) ölçümlerinden başlamak ve sistemin içinde gerçekte neler olup bittiğinin ayrıntılarına girmeden bunlar arasında matematiksel bir ilişki belirlemeye çalışmaktır. Sistem tanımlama da white-box, grey-box ve black-box olmak üzere üç temel model vardir. Sistem ile ilgili derin bilgi gerektiren durumlarda white-box, tanımlama sürecinde sistem ile ilgili bazı ön bilgiler kullanıldığında grey-box ve sistem için önceden bir bilgi kullanılmadan yalnızca süreçten elde edilen deneysel zaman serilerine dayalı olarak da black-box modeli kullanılır (Cavazzana, Barbosa, Torres ve Martinez, 2007).

\subsection{Simülasyon Devresi}

Düşürücü dönüştürücü devresi tasarlarken dönüştürücünün sabit durumda olduğunu varsayıp yarı iletken anahtarlama elemanını ideal kabul ederek endüktif ve kapasitif elemanların iç kayıpları ihmal edilmiştir. Ayrıca dönüştürücünün sürekli iletim modunda çalıştığı varsayılacaktır.

Şekil 1' deki düşürücü dönüştürücü devresi için Sürekli İletim Modunda, giriş voltajı (Vs) ile çıkış voltajı (Vo) arasındaki ilişki denklem (3.3)' te verilmiştir;

$$
\mathrm{d}=\frac{\mathrm{v}_{0}}{\mathrm{v}_{\mathrm{S}}}=\frac{\mathrm{t}_{\mathrm{on}}}{\mathrm{T}}
$$

Denklemde d görev çevrimi, $t_{\text {on }}$ anahtarın iletim süresini ve $\mathrm{T}$ anahtarlama periyodunu ifade etmektedir. Anahtarlama periyodu $\mathrm{T}$ şu şekilde ifade edilebilir;

$$
\mathrm{T}=\frac{\mathrm{I}_{\mathrm{L}} \mathrm{V}_{\mathrm{S}}}{\mathrm{V}_{0}\left(\mathrm{~V}_{\mathrm{S}}-\mathrm{V}_{0}\right)}
$$

Devredeki minimum endüktans ve kapasitans değerlerinin hesaplanabilmesi için aşağıdaki eşitlikler kullanılır;

$L=\frac{(1-D) R}{2 f}$

$\mathrm{C}=\frac{1-\mathrm{D}}{8 \mathrm{Lf}^{2}} * \frac{1}{\left(\frac{\Delta V_{0}}{V_{0}}\right)}$

Söz konusu eşitliklerde değerler yerine konulursa;

$\mathrm{L}=\frac{(1-\mathrm{D}) \mathrm{R}}{2 \mathrm{f}}=\frac{(1-0.417) 6.8}{2 \times 10000}=0.198 \mathrm{mH} \cong 0.2 \mathrm{mH}$

$\mathrm{C}=\frac{1-\mathrm{D}}{8 \mathrm{Lf}^{2}} * \frac{1}{\left(\frac{\Delta V_{0}}{V_{0}}\right)}=\frac{(1-0.417)}{8 \times 0.2 \times 10^{-3} \times\left(10000^{2}\right) * 0.01} \cong 400 \mu \mathrm{F}$

Düşürücü dönüştürücü devresinde giriş/çıkış değerleri 12/5V ve Anahtarlama frekansı $10 \mathrm{kHz}$ üzerinden çıkış gerilim dalgalanmasına göre gerekli hesaplamalar yapılmış ve devre elemanlarına ait değerler Tablo 1 üzerinde gösterilmiştir.

Tablo 1. Düşürücü Dönüştürücü Devre Parametreleri

\begin{tabular}{|c|c|c|}
\hline $\begin{array}{c}\text { Devre } \\
\text { Parametreleri }\end{array}$ & Açıklama & Değer \\
\hline$V_{s}$ & Giriş gerilimi & $12 \mathrm{~V}$ \\
\hline $\mathrm{L}$ & Endüktans değeri & $0.2 \mathrm{mH}$ \\
\hline $\mathrm{C}$ & Kapasitans değeri & $400 \mu \mathrm{F}$ \\
\hline $\mathrm{R}$ & Yük direnci & $6.8 \Omega$ \\
\hline$f_{s}$ & Anahtarlama frekansı & $10 \mathrm{kHz}$ \\
\hline$V_{0}$ & Çıkış gerilimi & $5 \mathrm{~V}$ \\
\hline $\mathrm{D}$ & Doluluk oranı & 0.417 \\
\hline$\% \Delta V_{0}$ & $\begin{array}{c}\text { Çıkış gerilimi } \\
\text { dalgalanma yüzdesi }\end{array}$ & 0.01 \\
\hline
\end{tabular}

DA-DA düşürücü simülasyon devresi tasarımı için Matlab/Simulink uygulaması kullanılmıştır. Kontrolsüz olarak tasarlanan referans simülasyon devresi Şekil 4'te gösterilmiştir.
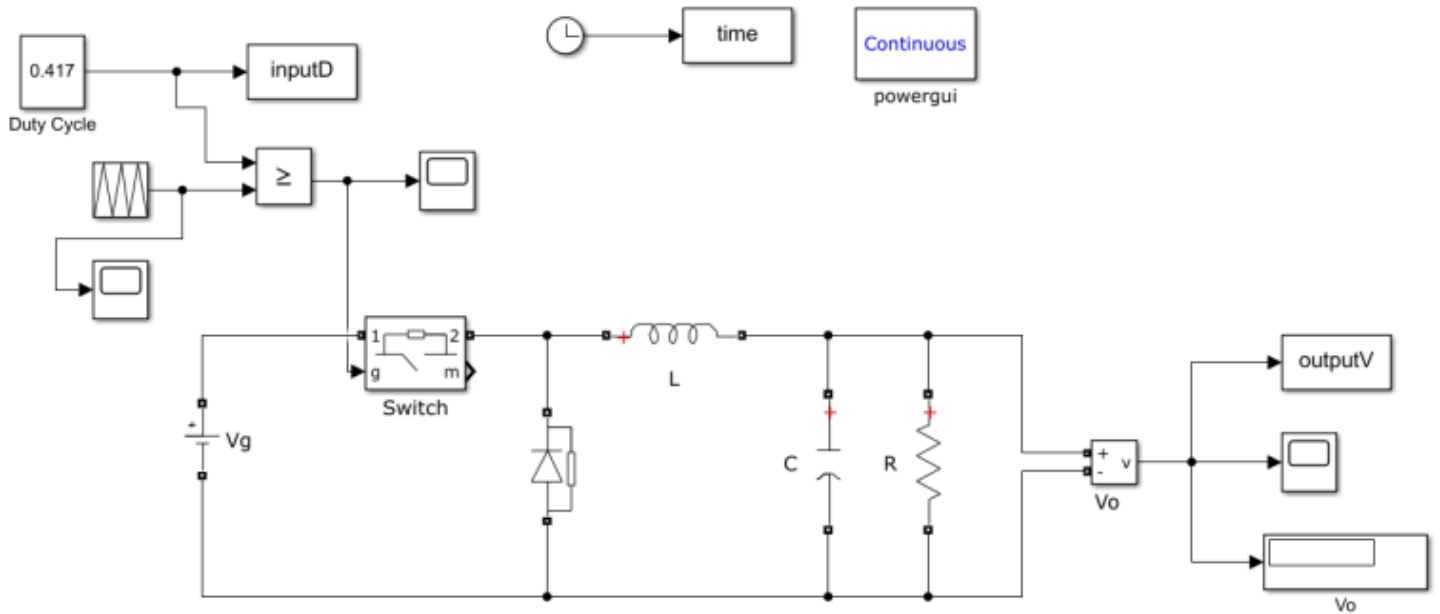

Şekil 4. Referans Simülasyon Devresi 
Referans simülasyon devresinin çıkış gerilimine ait grafikler Şekil 5'de verilmiştir.

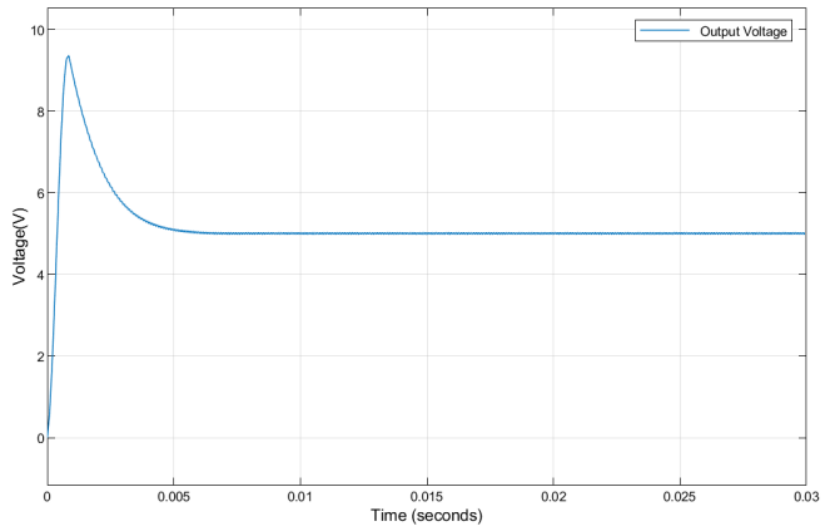

a)

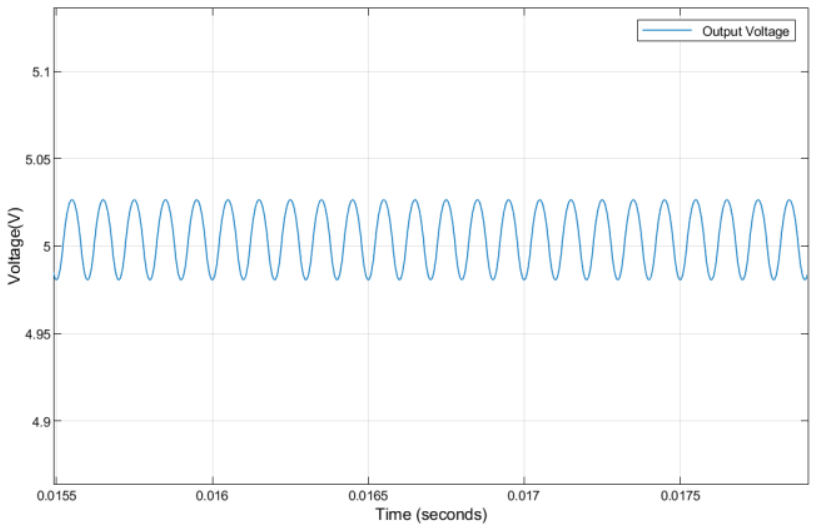

b)

Şekil 5. Referans Simülasyon Devresi Çıkış Gerilim Grafiği a) normal görüntü, b)yakınlaştırılmış görüntü

Şekil 5'deki çıkış grafiklerinden görüleceği üzere aşım miktarı \%90 seviyelerinde olduğu için istenmeyen durum meydana gelmiştir. Dolayısıyla sistem kontrole ihtiyaç duymaktadır. Sistemi iyileştirmek için sisteme gerilim modlu kapalı çevrim geri besleme ile PID kontrolör uygulanacaktır. Ancak PID kontrolör eklenmeden önce sistemin transfer fonksiyonunun çıkarılması gerekmektedir. Transfer fonksiyonu hesaplanırken Black-box sistem tanımlama metodu kullanılarak, en yüksek performanslı transfer fonksiyonu seçimi yapılmıştır. Şekil 6' da düşürücü dönüştürücü devre tasarımı aşamasından başlayıp sistemin en uygun transfer fonksiyonunu tahmin eden iş akış şeması verilmiştir.

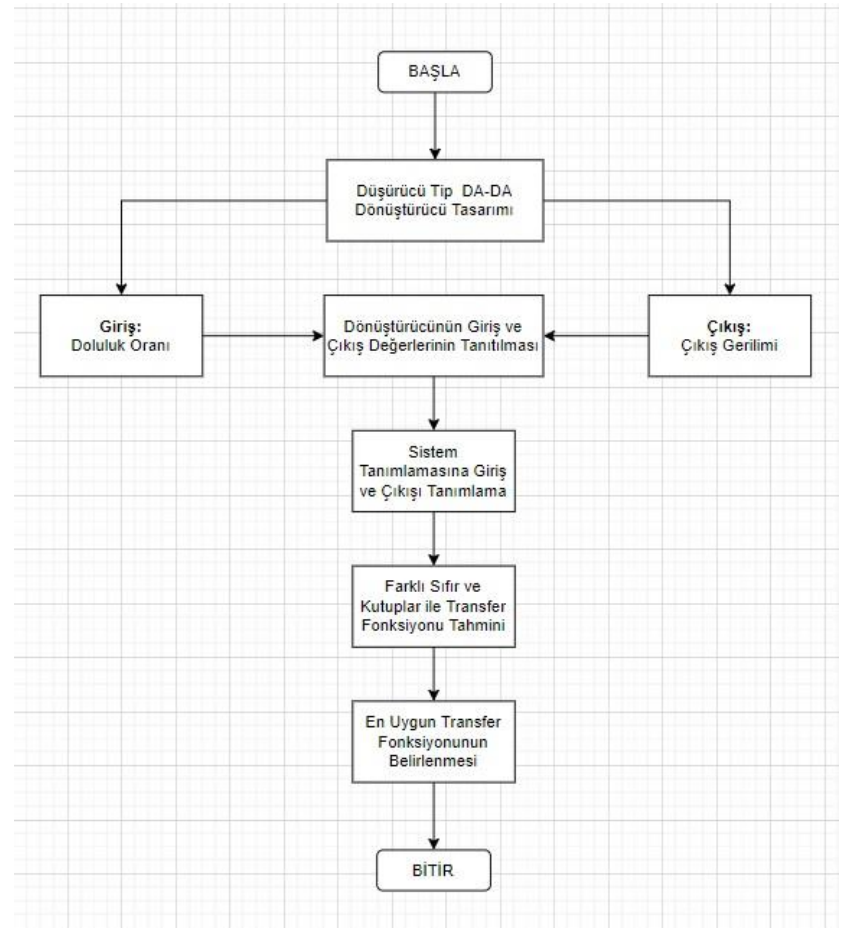

Şekil 6. Transfer Fonksiyonu Tahminini Açıklayan İş Akış Şemas1

Düşürücü dönüştürücü referans simülasyon devresinden alınan giriş ve çıkış değerleri Matlab Workspace ortamına aktarımı yapıldıktan sonra Matlab Sistem Identification Toolbox ile değişik pay ve payda değerleri seçilerek transfer fonksiyonları ve performans değerleri çıkarımı yapılmıştır. Burada, 2 kutup 1 sifirlı (tf1), 3 kutup 1 sifirlı (tf2), 3 kutup 2 sifirlı (tf3), 4 kutup 1 sifirlı (tf4) ve 5 kutup 1 sifirlı (tf5) transfer fonksiyonlarının model tahminleri yapılmıştır. Tablo 2 üzerinde tahminleri yapılan transfer fonksiyonlarının doğruluk yüzdeleri gösterilmektedir. 
Tablo 2. Farklı Kutup ve Sıfır Değerli Transfer Fonksiyonları ve Doğruluk Yüzdeleri

\begin{tabular}{|c|c|c|c|c|}
\hline $\begin{array}{c}\text { Transfer } \\
\text { Fonksiyon } \\
\mathbf{u}\end{array}$ & $\begin{array}{c}\text { Kutu } \\
\mathbf{p}\end{array}$ & Sifır & Model & $\begin{array}{c}\text { Doğruluk } \\
(\%)\end{array}$ \\
\hline $\mathrm{tf5}$ & 5 & 1 & $\frac{5.195 \mathrm{e}-05 \mathrm{~s}^{2}+2.315 \mathrm{e}-07 \mathrm{~s}}{2}$ & 92.02 \\
\hline $\mathrm{tf} 4$ & 4 & 1 & $\frac{\mathrm{s}^{5}+0.2009 \mathrm{~s}^{4}+0.02122 \mathrm{~s}^{3}+0.000724 \mathrm{~s}^{2}+7.283 \mathrm{e}-06 \mathrm{~s}+1.932 \mathrm{e}-08}{\mathrm{~s}^{4}+0.09415 \mathrm{~s}^{3}+0.006278 \mathrm{~s}^{2}+0.0001612 \mathrm{~s}+1.049 \mathrm{e}-06}$ & 97.30 \\
\hline $\mathrm{tf} 3$ & 3 & 2 & $\frac{0.003276 \mathrm{~s}+1.258 \mathrm{e}-05}{\mathrm{~s}^{3}+0.07173 \mathrm{~s}^{2}+0.00229 \mathrm{~s}+1.452 \mathrm{e}-05}$ & 93.86 \\
\hline $\mathrm{tf} 2$ & 3 & 1 & $\frac{0.04747 \mathrm{~s}+0.000174}{\mathrm{~s}^{3}+0.07173 \mathrm{~s}^{2}+0.00229 \mathrm{~s}+1.452 \mathrm{e}-05}$ & 93.86 \\
\hline $\mathrm{tf} 1$ & 2 & 1 & $\frac{0.6058 \mathrm{~s}+0.002693}{\mathrm{~s}^{2}+0.2704 \mathrm{~s}+0.0002239}$ & 80.46 \\
\hline
\end{tabular}

Tablo 2' de tf4 olarak adlandırılan 4 kutuplu 1 sifirlı sistemin transfer fonksiyonu doğruluk yüzdesi $\% 97.3$ olarak belirlenmiştir. Yüksek oranda doğruluk yüzdesine sahip olan tf4 fonksiyonu aynı zamanda bundan sonraki süreçte referans alınacak fonksiyon olarak belirlenmiştir.

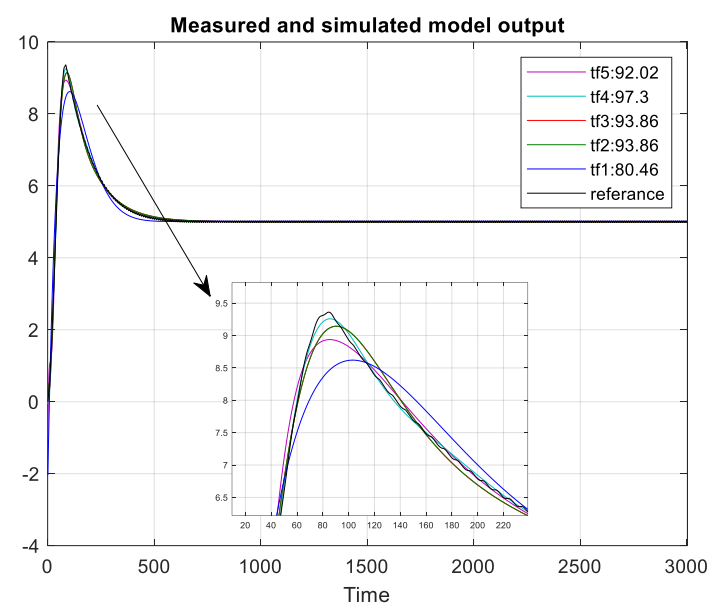

Şekil 7. Farklı Transfer Fonksiyonlarının Basamak Cevapları

Düşürücü dönüştürücü referans simulink devresinin açık çevrim blok diyagramı Matlab/Simulink ortamında modellenmesi Şekil 8' de görülmektedir.

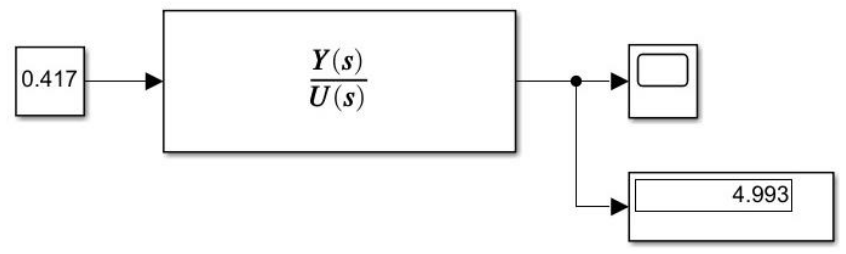

Şekil 8. Transfer Fonksiyonu Açık Çevrim Cevabı

Referans transfer fonksiyonu olarak kullanılacak (tf4) fonksiyonunun pay ve payda değerleri Şekil 8 içerisindeki transfer fonksiyonu bloğuna aktarılmıştır. Şekil 9' da transfer fonksiyonunun açık çevrimdeki çıkış geriliminin grafiği verilmiştir.

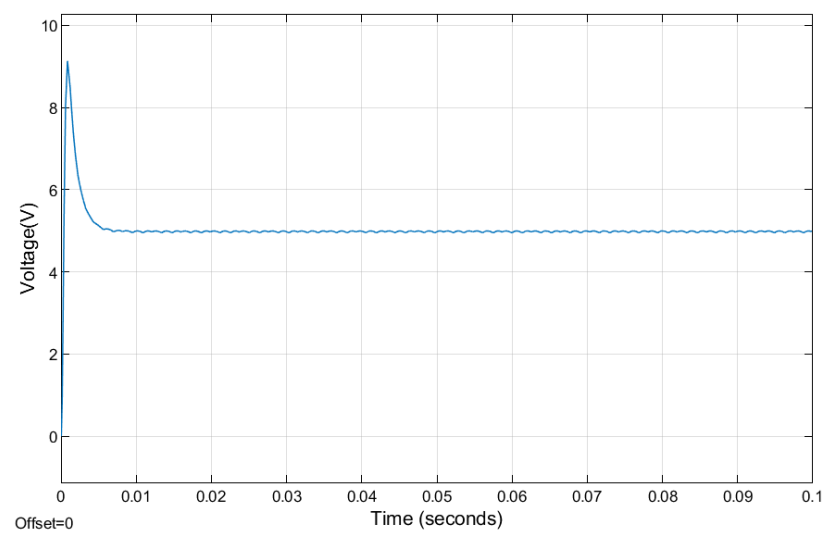

Şekil 9. Transfer Fonksiyonu Açık Çevrim Çıkış Grafiği

Tablo 3'te ise açık çevrim cevabının performans değerleri verilmiştir.

Tablo 3. Açık Çevrim Cevabı Performans Değerleri

\begin{tabular}{|c|c|}
\hline Parametre & Kontrolsüz Cevap \\
\hline Aşım Yüzdesi (\%) & 84.26 \\
\hline Yerleşme Zamanı (ms) & 5.063 \\
\hline Yükselme Zamanı (ms) & 0.24 \\
\hline Tepe Değeri (V) & 9.13 \\
\hline Tepe Zamanı (ms) & 0.86 \\
\hline
\end{tabular}

Tablo 3 değerleri sistemin aşım yüzdesinin yüksek olduğunu ve kontrole ihtiyaç duyduğunu göstermektedir. Düşürücü dönüştürücü devresine PID kontrolör eklenerek sistem analizi yapılmıştır. 


\subsection{PID Kontrolör Uygulaması ve Simülasyon Sonuçları}

Düşürücü dönüştürücü referans devresindeki aşım ve salınım miktarlarını azaltmak ve sistemi daha kararlı hale getirebilmek için devreye geri beslemeli, gerilim modlu PID kontrolör eklenmiş ve simülasyon devresi yeniden tasarlanmıştır. Matlab/Simulink ortamında tasarlanan düşürücü dönüştürücü devresi Şekil 10' da görülmektedir.

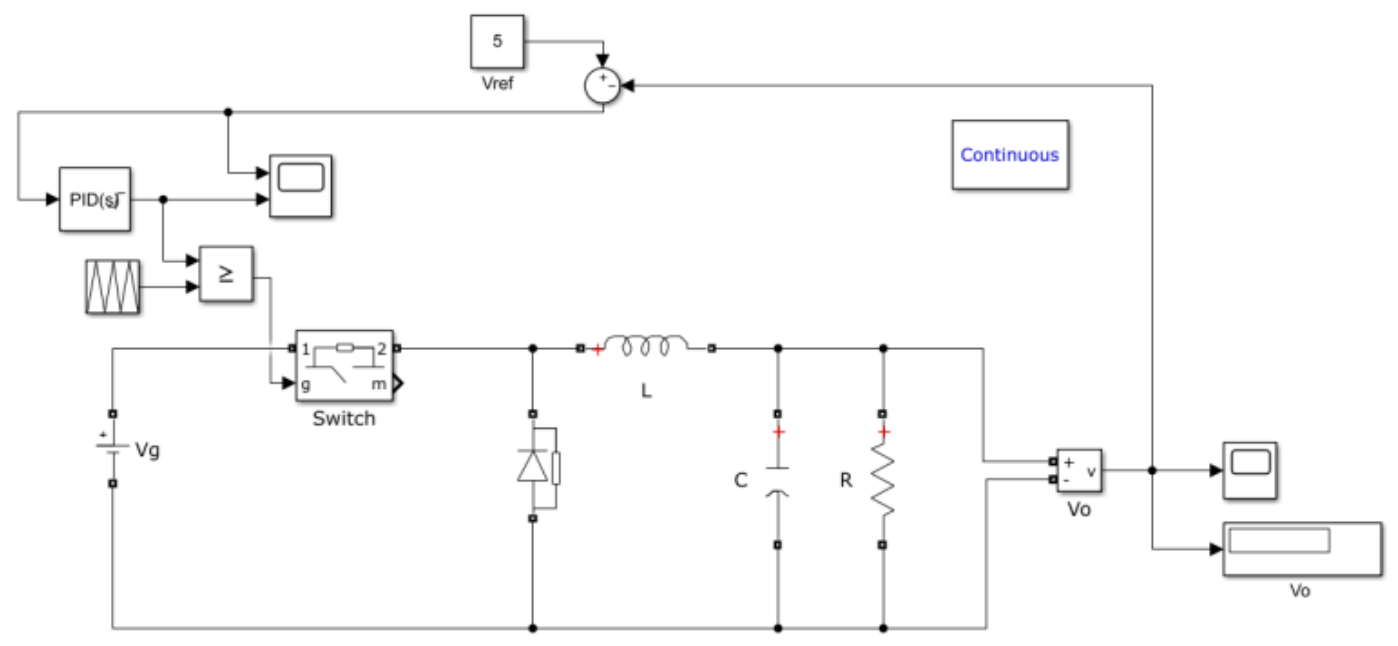

Şekil 10. PID Kontrolör ile Düşürücü Dönüştürücü Devresi

Düşürücü dönüştürücü referans simulink devresine PID kontrolör eklendikten sonra PID kontrolörün kontrol parametreleri ayarlanarak sisteme tanımlanması gerekmektedir.

PID Kontrolörün kontrol parametrelerini bulmak için Matlab kod ortamındaki fonksiyonlardan yararlanılmıştır (Marti, Bradu ve Vinuela, 2015). Düşürücü dönüştürücü referans simulink devresinden alınan giriş ve çıkış değişkenleri Matlab Workspace ortamına aktarılıp ardından sistem identification toolbox üzerinde transfer fonksiyonu tahmini yapilarak PID kontrol parametrelerine erişim sağlanmıştır.

Şekil 11' de PID kontrolör eklendikten sonra çıkış gerilimi grafiği gösterilmiş̧ir.

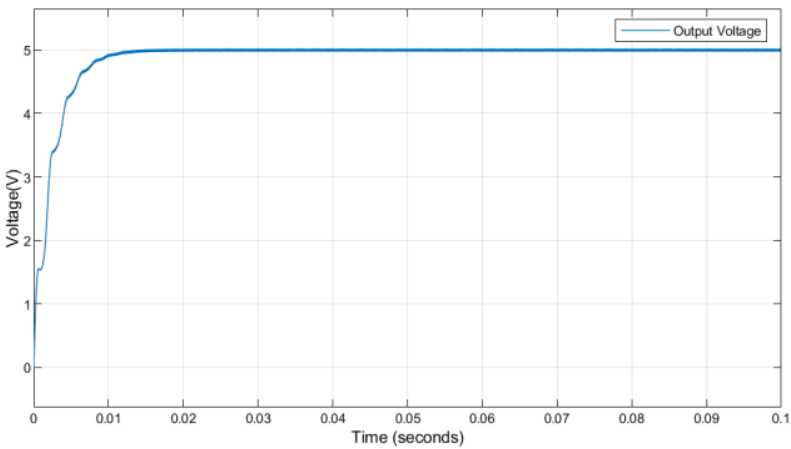

a)

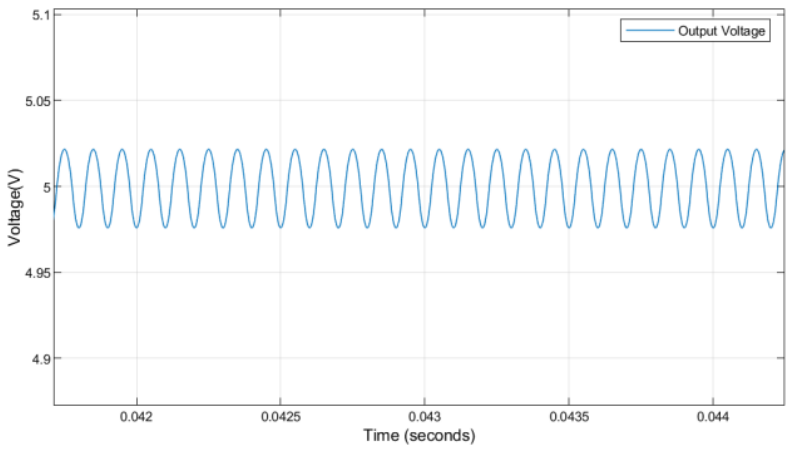

b)

Şekil 11. PID Kontrolörlü Düşürücü Dönüştürücü Devresi Çıkış Gerilim Grafiği a) normal görüntü, b)yakınlaştırılmış görüntü

Şekil 11'de görüleceği üzere PID kontrolör parametre değerleri ayarlandıktan sonra sistemdeki aşım seviyesi azaltılmış ve sistem daha kararlı bir hale getirilmiştir. PID kontrolörlü düşürücü dönüştürücü simülasyon devresinin kapalı çevrim blok diyagramı Şekil 12' de görülmektedir.

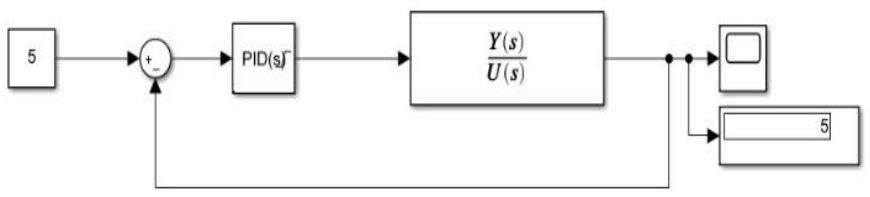

Şekil 12. PID Kontrollü Transfer Fonksiyonu Kapalı Çevrim Cevab1

Blok diyagrama ait cevap grafiği Şekil 13' de gösterilmiştir. 


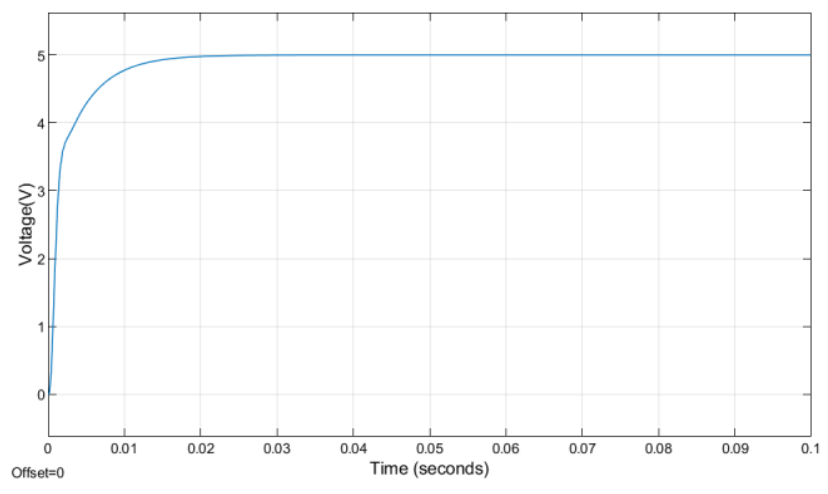

Şekil 13. PID Kontrollü Transfer Fonksiyonu Kapalı Çevrim Cevap Grafiği

Tablo 4'te kontrolsüz sistem ile PID kontrollü sistemin performans değerleri karşılaştırılmıştır.

Tablo 4. Kontrolsüz Cevap ile PID Kontrollü Cevapların performans kıyaslaması

\begin{tabular}{|c|c|c|}
\hline Parametre & $\begin{array}{c}\text { Kontrolsüz } \\
\text { Cevap }\end{array}$ & $\begin{array}{c}\text { PID Kontrollü } \\
\text { Cevap }\end{array}$ \\
\hline Aşım Yüzdesi (\%) & 84.26 & 0.463 \\
\hline Yerleşme Zamanı & $5.06 \mathrm{~ms}$ & $11.45 \mathrm{~ms}$ \\
\hline Yükselme Zamanı (ms) & $0.24 \mathrm{~ms}$ & $5.92 \mathrm{~ms}$ \\
\hline Tepe Değeri (V) & 9.13 & 5 \\
\hline Tepe Zamanı (ms) & $0.86 \mathrm{~ms}$ & $0.1 \mathrm{~s}$ \\
\hline
\end{tabular}

Tablo 4'te görüleceği üzere kontrolsüz olan düşürücü dönüştürücü referans devresine PID kontrolör eklendikten sonra aşım miktarının neredeyse sıfıra düştüğü ve kontrolörün sistem performansını iyileştirdiği görülmüştür.

\section{SONUÇ}

Sistemlerin matematiksel olarak modellenmesi uzun ve dikkatli hesaplamalar gerektiren bir işlemdir. Sistem tanımlama metotları bu noktada matematiksel modelleme gerektiren işlemleri çok hızlı ve doğru bir şekilde çözmemize olanak sağlamaktadır.

Bu çalışmada, bir DA-DA düşürücü tip dönüştürücü için yeni bir modelleme tasarımı amaçlanmıştır. Tasarım için giriş/çıkış değerleri tanıtılmış Black-box sistem tanımlama yöntemi kullanılmıştır. Burada, tasarımı yapılan sistemin transfer fonksiyonunun katsayıları belirlenmiştir. Katsayılar belirlenirken, önerilen modellerin performans değerleri ve sisteme hangi oranda benzediği ortaya konmuştur. Önerilen transfer fonksiyonunun performansı değerlendirildiğinde \%97.3 oranında benzerlik elde edilmiştir. Tasarıma ait simülasyon devresinin aşım miktarını azaltmak ve sistemi daha kararlı bir hale getirmek için devreye PID kontrolör eklenerek kontrolör parametreleri belirlenmiştir. Elde edilen transfer fonksiyonunun asıl sisteme olan benzerliğini ölçmek amacıyla transfer fonksiyonunun kontrollü ve kontrolsüz durumlardaki açık çevrim ve kapalı çevrim performansları kıyaslanmıştır. Devre sonuçları incelendiğinde, Black-box sistem tanımlama yöntemi kullanılarak tasarımı yapılan sistemin transfer fonksiyonunun devreyi hızlı ve doğru bir şekilde modelleyebildiği görülmüştür. Buna göre çalışmanın içeriği ve sonuçları güç elektroniğinde ve çeşitli alanlarda önemli bir katkı sağlama potansiyeline sahip olduğu görülmüştür

\section{Kaynakça}

Abbas, N., \& Abbas , G. (2018). Estimating Dynamics of Switching Converters Using System Identification Technique. Pakistan Journal of Engineering and Technology, PakJET, 21-28.

Algreer, M., Armstrong, M., \& Giaouris, D. (2009). System identification of PWM dc-dc converters during abrupt load changes. 2009 35th Annual Conference of IEEE Industrial Electronics (s. 1788-1793). Porto: IEEE.

Andries, V. D., Goras, L., David, E., Buzo, A., \& Pelz, G. (2019). On the Accuracy of a Time-Domain Identification Method for a DC-DC Buck Converter. 2019 International Symposium on Signals, Circuits and Systems (ISSCS) (s. 16). Lasi, Romanya: IEEE.

Bodur, H. (2014). Güç Elektroniği 3. Baskı (s. 179-248). içinde İstanbul, Türkiye: Birsen Yayınevi.

Buiatti, G. M., Amaral, A. R., \& Marques Cardoso, A. J. (2007). "Parameter Estimation of a DC/DC Buck converter using a continuous time model" in Power Electronics and Applications,. 2007 European Conference, s. 1-8.

Cavazzana, E., Barbosa, B. H., Torres, L. A., \& Martinez, C. B. (2007). COMPARISON OF WHITE-BOX AND BLACKBOX MODELS OF A REALHYDRAULIC PUMPING SYSTEM USING A VARIABLE SPEED DRIVE. 19th International Congress of Mechanical Engineering. Brasilia.

Cen, Z., \& Stewart, P. (2017). Condition Parameter Estimation for Photovoltaic Buck Converters Based on Adaptive Model Observers. IEEE Transactions on Reliability , s. 148-160.

Choi, J. C. (1998). System Identification of Power Converters Based on a Black-Box Approach. IEEE Transactions on Circuits and Systems, I: Fundamental Theory and Applications, Vol. 45, No. 11.

Emadi, A. (2005). Handbook of Automotive Power Electronics and Motor Drives 1st ed. Florida, USA: Taylor\&Francis.

Goodwin, G. C. (1977). Dynamic System Identification: Experiment Design and Data Analysis. Academic Press.

Jin-Hong, J., Tae-Jin, K., Kwang-Su, K., \& Kwang-Hwa, K. (2004). Parameter Estimation by OE model of DC-DC Converter System for Operating Status Diagnosis. KIEE International Transaction on Electrical Machinery and Energy Conversion Systems, (s. 206-210).

Li, B. X., \& Low, K. S. (2016). Low Sampling Rate Online Parameters Monitoring of DC-DC Converters for Predictive-Maintenance Using Biogeography-Based Optimization. in IEEE Transactions on Power Electronics (s. 2870-2879). IEEE .

Marti, R., Bradu, B., \& Vinuela, E. B. (2015). PID_TUNE: A PID Autotuning Software Tool On Unicos CPC. Proceedings of ICALEPCS2015. Melbourne, Australia.

Mathworks. https://www.mathworks.com/help/ident/gs/identify-linearmodels-using-the-gui.html

Osman, S. \&. (2011). Load Identification of DC-DC converter. Electrical Engineering Master Thesis, Blekinge Institute of Technology. 
Osman, S., \& Osman, S. (2011). Load Identification of DC-DC converter. This thesis is presented as part of Degree of Master of Science in Electrical Engineering. Blekinge Institute of Technology.

Özdemir, Ö., \& Yazıcı, İ. (2018). Ayrık Zaman KKK, LQR ve PID Kontrolör Performanslarının Alçaltıcı Tip Da-Da Dönüştürücü Üzerinde Kıyaslanması. Sakarya Üniversitesi Fen Bilimleri Dergisi, 8-15.

Padhee, S., Pati, U. C., \& Mahapatra, K. (2018). Closed-loop parametric identification of DC-DC converter. Proceedings of the Institution of Mechanical Engineers, Part I: Journal of Systems and Control Engineering, 1429-1438.

Pembegüllü, İ. (2019). Optimized PID Control Of DC-DC Buck Converter,. Düzce University, Graduate School of Natural and Applied Sciences, Department of Electrical Electronics and Computer Engineering, Master's Thesis.

Rashid, M. H. (2007). Power Electronics Handbook 3rd ed. Florida, USA.

Taborda, J. A., Angulo, F., \& Olivar, G. (2011). Estimation of parameters in Buck converter with Digital-PWM control based on ZAD strategy. 2011 IEEE Second Latin American Symposium on Circuits and Systems (LASCAS) (s. 1-4). Bogota, Colombia: IEEE.

Yalduz, H. (2015). DA-DA DÜŞÜREN DÖNÜŞTÜRÜCÜ TASARIMI VE PI KONTROLÜ. Yüksek Lisans Tezi. Van: YÜZÜNCÜ YIL ÜNIVERSITESI FEN BILIMLERI ENSTITÜSÜ.

Yalduz, H. (2015). Design and PI Control of DC-DC Buck Converter. Van: Yüzüncü Y1l University: Institute of Science and Technology.

Zhao, Z. (2008). Design and Practical Implementation of Digital Auto-Tuning and Fast-Response Controllers for Low-Power Switch-Mode Power Supplies. A thesis submitted in conformity with the requirements for the degree of Doctor of Philosophy. Canada: Graduate Department of Electrical and Computer Engineering University of Toronto. 